\title{
Evaluating damage in optical elements using an amplified spontaneous emission beam
}

\author{
Qiong Zhou ${ }^{1,2}$, Jiangfeng Wang ${ }^{1}$, Yajing Guo ${ }^{1}$, Dean Liu ${ }^{1}$, and Jianqiang Zhu ${ }^{1}$ \\ ${ }^{1}$ National Laboratory on High Power Laser and Physics, Shanghai Institute of Optics and Fine Mechanics, Chinese Academy of \\ Sciences, Shanghai 201800, China \\ ${ }^{2}$ University of Chinese Academy of Science, Beijing 100049, China
}

(Received 11 September 2015; revised 21 October 2015; accepted 2 November 2015)

\begin{abstract}
A method to evaluate damage in optical elements with the near field of an amplified spontaneous emission (ASE) beam has been developed. Local peak intensities are generally distributed randomly in the near field of a laser beam. The partial coherence of the ASE source results in a very smooth beam profile. The coherence time of ASE is much less than the pulse width. Small-scale intensity modulations can be smoothed out rapidly within the time of a pulse width. In the experiments, ASE is generated from a multifunctional high-performance Nd:glass system, with a pulse duration of $3 \mathrm{~ns}$, a spectral width (full width at half maximum, FWHM) of $1 \mathrm{~nm}$ and an adjustable energy range from 1 to $10 \mathrm{~J}$. The damage thresholds of samples induced by ASE are two to three times higher than those induced by a laser with the same size of test spot. Furthermore, the ASE beam has great potential for the detection of defects over a large area and the conditioning of optical elements.
\end{abstract}

Keywords: amplified spontaneous emission; damage threshold test; partially coherence; uniform intensity distribution

\section{Introduction}

Accurately predicting the lifetime of optics in high-power fusion lasers is very important in managing laser facilities ${ }^{[1-3]}$. The defects randomly distributed in optical elements and the light spot which irradiates the sample surface together determine the damage threshold ${ }^{[4]}$. As is well known, a large-area spot and a smooth intensity distribution can reveal more defects, which are the major factors for an accurate damage measurement. The far field of the test spot is usually used because of its smooth Airy pattern, in which a majority of the energy is concentrated in the main lobe. But the beam size is very small. When a 1-to- 1 test is used, there is a clear dependence on the beam size, and not all the defects are revealed ${ }^{[5]}$. If a raster-scan test is used, the beam shape - Gaussian or flat top - needs to be considered, because of beam overlap. Also scanning times are very long with small size beams ${ }^{[5]}$. Hence, a test beam in the near field would seem to be a better choice, because a large size spot can be acquired. A laser beam is usually used as the irradiation source in the laserinduced damage threshold (LIDT) test. But, during laser propagation through the laser system, the near-field intensity

Correspondence to: J. Zhu, No. 390, Qinghe Rd., Jiading, Shanghai 201800, China. Email: jqzhu@siom.ac.cn distribution is strongly influenced by random modulation of both the phase and amplitude. Local peaks of intensity result, randomly distributed in the near field. Combined with the defects of the sample, nonuniformity of the laser source makes the damage distribution more complicated to determine. If the intensity distribution on the sample surface was uniform, then damage would be most likely to occur in the areas where defects in the samples were located. Thus, the damage distribution would correspond to that of the defects. Compared to a laser source, a light source with a uniform intensity distribution should be more suitable to test damage thresholds and derive directly the damage characteristics induced by defects in samples.

Amplified spontaneous emission (ASE) is one typical kind of partially coherent light $(\mathrm{PCL})^{[6,7]}$. The coherence time of ASE is far shorter than the pulse duration due to temporal incoherence. There is less spatial modulation during ASE beam propagation to the sample surface, leading to a reduction in intensity fluctuations in the irradiated spot on the sample. Therefore, ASE could be a preferable irradiation source in the damage threshold test. Furthermore, if the output energy of ASE from the experimental installation were sufficiently high, a test spot with a large size could be acquired and the test time could also be shortened.

In this letter, a method using an ASE-induced damage threshold test is proposed and validated. The ASE beam is 


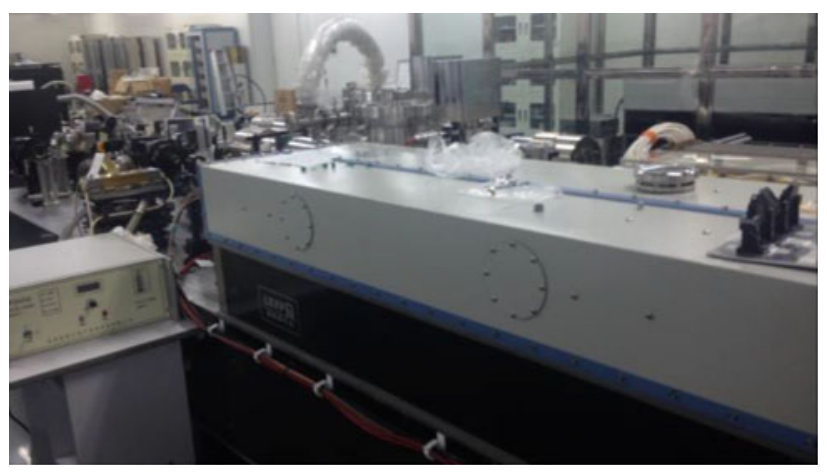

Figure 1. Multifunctional high-performance Nd:glass system based on a four-pass amplifier.

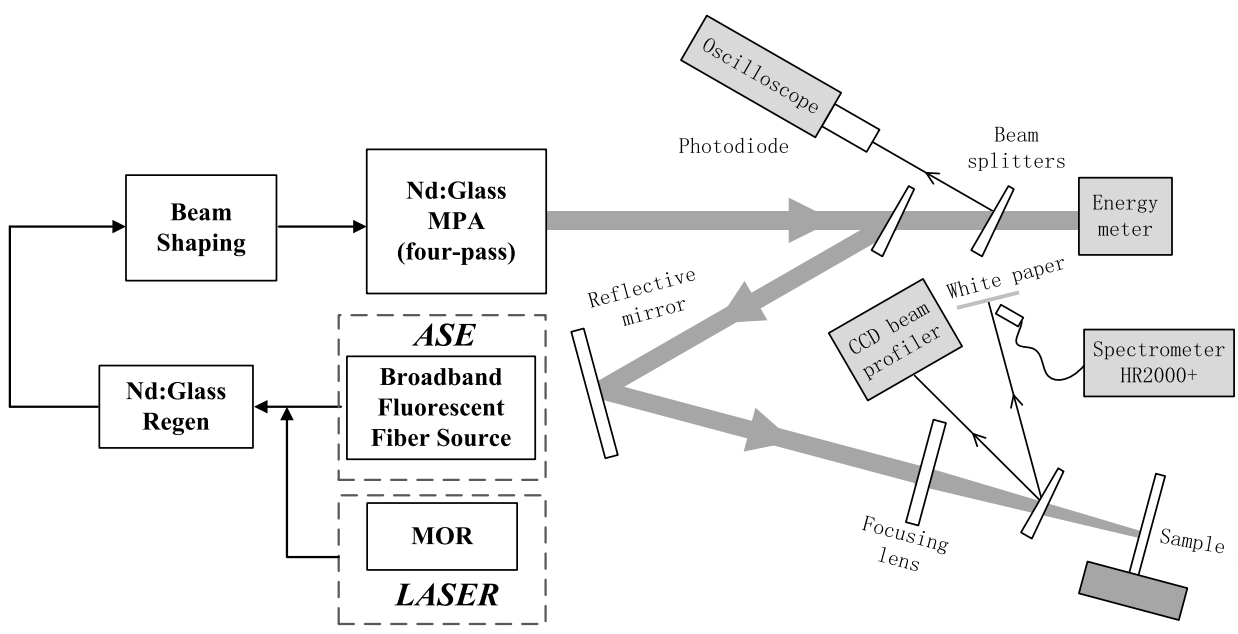

Figure 2. Schematic of a damage threshold testing facility using the ASE beam and the laser beam respectively.

generated from a multifunctional high-performance Nd:glass system based on a four-pass amplifier (Figure 1) with a certain spectral width and a smooth intensity distribution. The energy of the ASE can reach $10 \mathrm{~J}$ or more. The intensity distribution of the ASE and the laser are compared. Three kinds of samples are tested with this method, including $\mathrm{TiO}_{2}$ high-reflection optical film, fused silica glass and $\mathrm{K} 9$ glass. Their ASE-induced damage threshold (AIDT) is clearly two to three times higher than when tested using a laser beam with the same size of test spot, leading to an accurate evaluation of the damage threshold.

\section{Experiments}

\subsection{Experimental setup and method}

The AIDT testing facility was built as shown in Figure 2. The broadband fluorescent source was coupled into multistage amplifier chains consisting of an $\mathrm{Nd}$ :glass regenerative amplifier, a beam shaping system and a four-pass amplifier. The spectral width of the ASE light was $4 \mathrm{~nm}$ (full width at half maximum, FWHM) because of gain narrowing in the Nd:glass rod. On passing through a narrow band filter, the final output spectral width was $1 \mathrm{~nm}$ (FWHM), as shown in Figure 3. An output energy of $10 \mathrm{~J}$ with a beam size of $18 \times 18 \mathrm{~mm}$ could be obtained, while the energy injected into the four-pass amplifier was about $1 \mathrm{~mJ}$. An optical shutter shaped the ASE light with an opening time of 3 ns (Figure 4). In the measurement setup, the ASE light was focused on the surface of the samples by a focusing lens with a diameter of $50 \mathrm{~mm}$ and a focal length of $1580 \mathrm{~mm}$. Samples, which were fixed on a three-dimensional translation stage, included $\mathrm{TiO}_{2}$ high-reflection film, fused silica glass and K9 glass. The optical distance between the sample and the focusing lens was fixed, leading to a square spot with a size of $0.914 \times$ $0.914(\mathrm{~mm})$. One shot was delivered every $15 \mathrm{~min}$, with an output energy level that could be varied from $1 \mathrm{~J}$ to more than $10 \mathrm{~J}$ by adjusting the power supply voltage. Therefore, the total fluence irradiated on the sample surface could be varied during the experiment. The spatial profile was recorded by a camera-based spot profiler and the temporal profile was recorded by a photocathode coupled to an oscilloscope.

According to the standard ISO-11254 for the determination of damage thresholds of optical surfaces, 1-on-1 measurement was adopted. One shot of ASE was radiated on each testing site of the sample surface. Then the light 


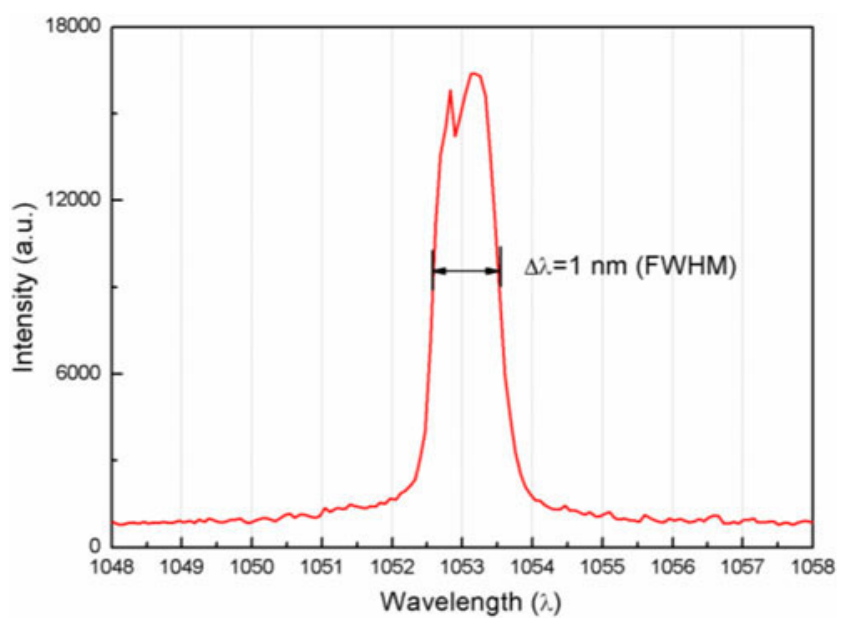

Figure 3. Spectrum profile of the ASE beam.

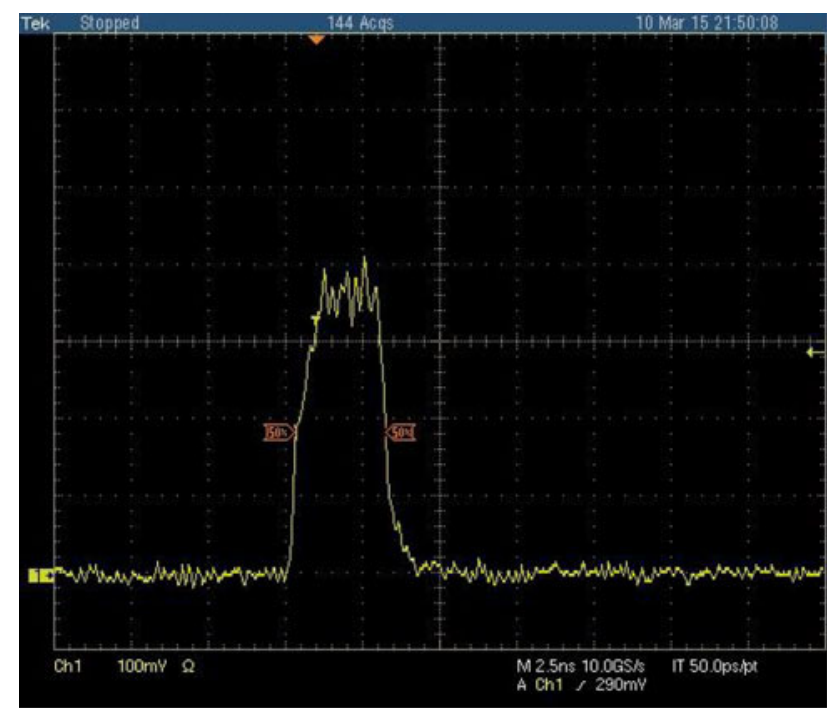

Figure 4. Temporal profile of the ASE beam.

spot was moved to the next testing site, no matter whether the previous site had been damaged or not. The energy density was gradually increased within the damage energy density range, and the damage morphologies at different energy densities were detected.

In order to assess any improvements in using the AIDT test method, a testing facility using LIDT was also built, as shown in Figure 2. The laser beam outputted from the main oscillator had a wavelength of $1053 \mathrm{~nm}$, a pulse duration of $3 \mathrm{~ns}$ and a spectral width (FWHM) of $10^{-3} \mathrm{~nm}$.

\subsection{Experimental results}

The output ASE and laser beam from this four-pass amplifier have flat-top profiles as shown. Because of the complete coherence of the laser light, interference, diffraction and nonlinear effects arise, which cause small-scale intensity
Table 1. Comparison of damage test results in samples.

\begin{tabular}{lll}
\hline Sample & LIDT $\left(\mathrm{J} / \mathrm{cm}^{2}\right)$ & AIDT $\left(\mathrm{J} / \mathrm{cm}^{2}\right)$ \\
\hline $\mathrm{TiO}_{2}$ & 4 & 12.5 \\
$\mathrm{SiO}_{2}$ & 14 & 30.5 \\
$\mathrm{~K} 9$ & 10.5 & 27.5 \\
\hline
\end{tabular}

perturbations. Thus, coherent fringes (marked by red box) and diffraction rings with a high contrast ratio are clearly observed in the intensity distribution of laser spot shown in Figures 5(b) and 5(h). The near-field fill factor of the laser beam is $52 \%$ and the modulation is 1.9. However, the ASE is PCL with a larger beam divergence angle and a broader spectral width of $4 \mathrm{~nm}$ (FWHM) after gain narrowing. The coherence time of the ASE is $3 \mathrm{ps}$, which is much less than the pulse width of 3 ns. Therefore, small-scale intensity modulations can be smoothed out rapidly within the time of a pulse width. There are no distinguishable coherence fringes observed in the intensity distribution of the ASE in Figures 5(a) and 5(g). In addition, diffraction patterns are found in the intensity distributions both of the ASE and laser spots, caused by the finite aperture of the optics. But, according to the fringe contrast of these diffraction patterns, the variations of the laser intensity distribution are apparently smaller in scale spatially than those of the ASE intensity distribution. This is due to PCL smoothing out the modulation to a certain extent. The near-field fill factor of the ASE is $60 \%$ and the modulation is 1.6.

The damage threshold is determined by the ratio of the damage area to the light spot area. Based on statistical analysis of the test results, the energy density which results in a ratio of zero is taken as the damage threshold of a sample. A curve relating the damage ratio to the energy density is nonlinearly fitted. The damage threshold of three kinds of samples measured using the ASE beam and the laser beam are shown in Figure 6 and Table 1.

The experimental results show that the AIDT is two to three times higher than the LIDT. The two main factors leading to this are the random defect distribution of the sample and random nonuniformities in the near field. During laser propagation, phase front distortion of the near field is caused by various sources of noise modulation, leading to large random intensity nonuniformities in the near field. For a fixed defect density in the samples, damage may occur first in the defect area due to nonuniformity of the test light spot. Thus, the damage threshold tested by a laser beam is much lower than the typical damage threshold of the samples. Therefore, under the joint action of defects and beam nonuniformity, which are randomly distributed, inaccuracy occurs in the evaluation of the laser damage resistance of the optical elements. However, nonuniformity of the intensity distribution can be suppressed by using PCL, such as ASE. The coherence time of ASE is far less than the pulse duration, due to spatial and temporal incoherence. 
(a)

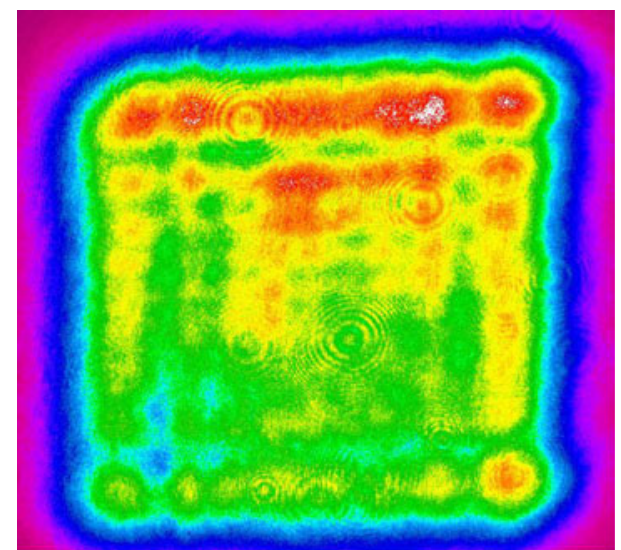

(c)

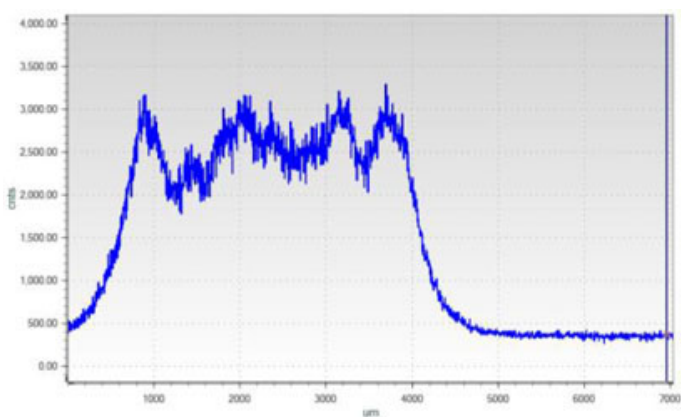

(e)

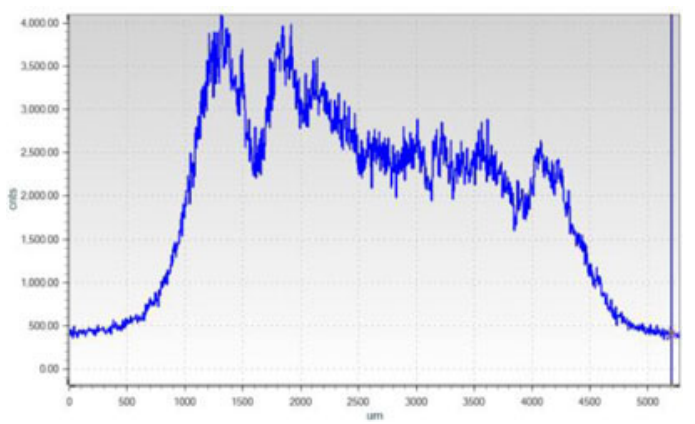

(g)

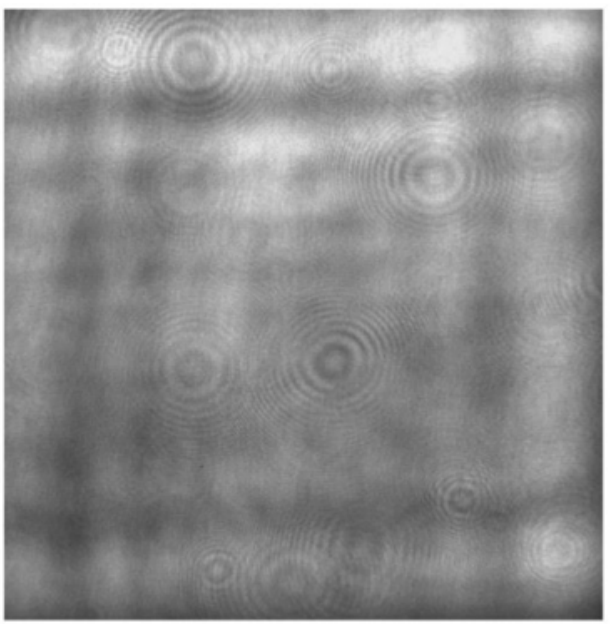

(b)

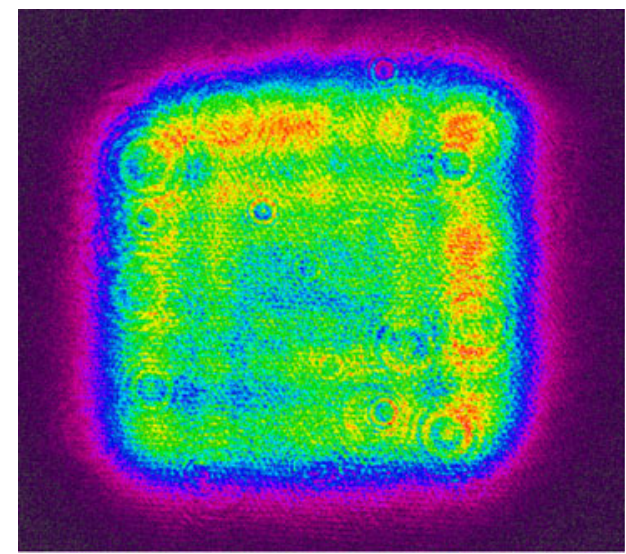

(d)

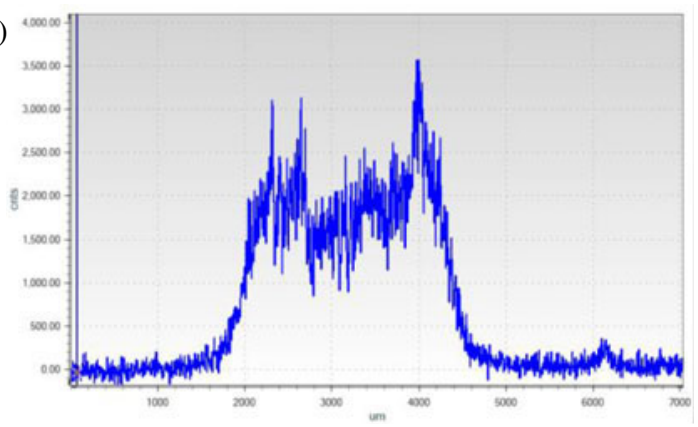

(f)

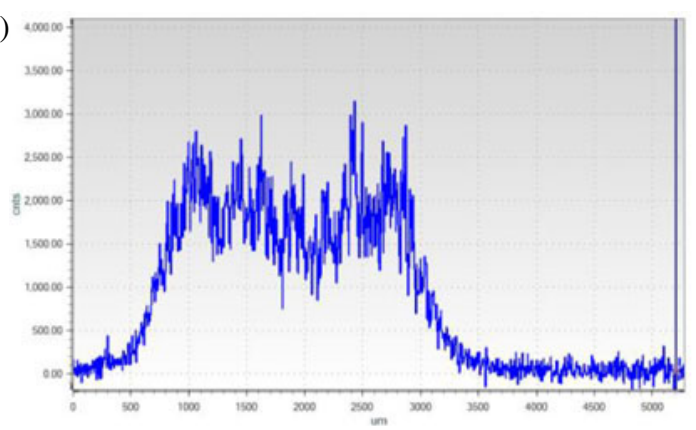

(h)

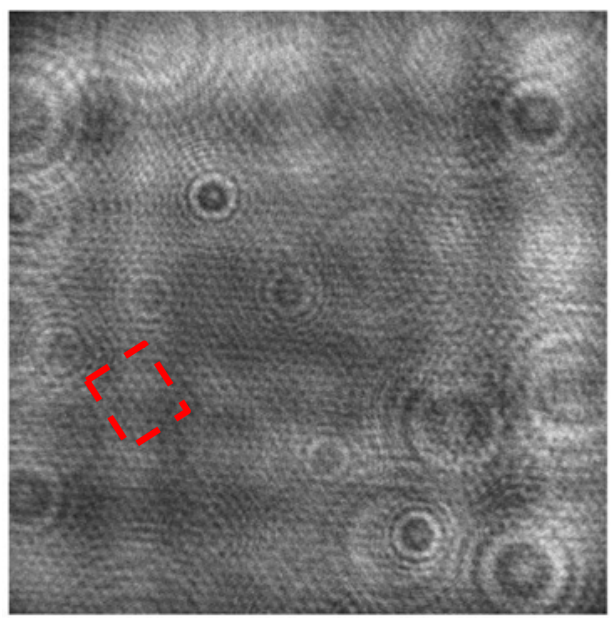

Figure 5. Two-dimensional intensity distribution of the ASE spot (a) and the laser spot (b); one-dimensional intensity distribution of the ASE spot (c), (e); and the laser spot (d), (f); enlarged view of the ASE spot (g) and the laser spot (h). 

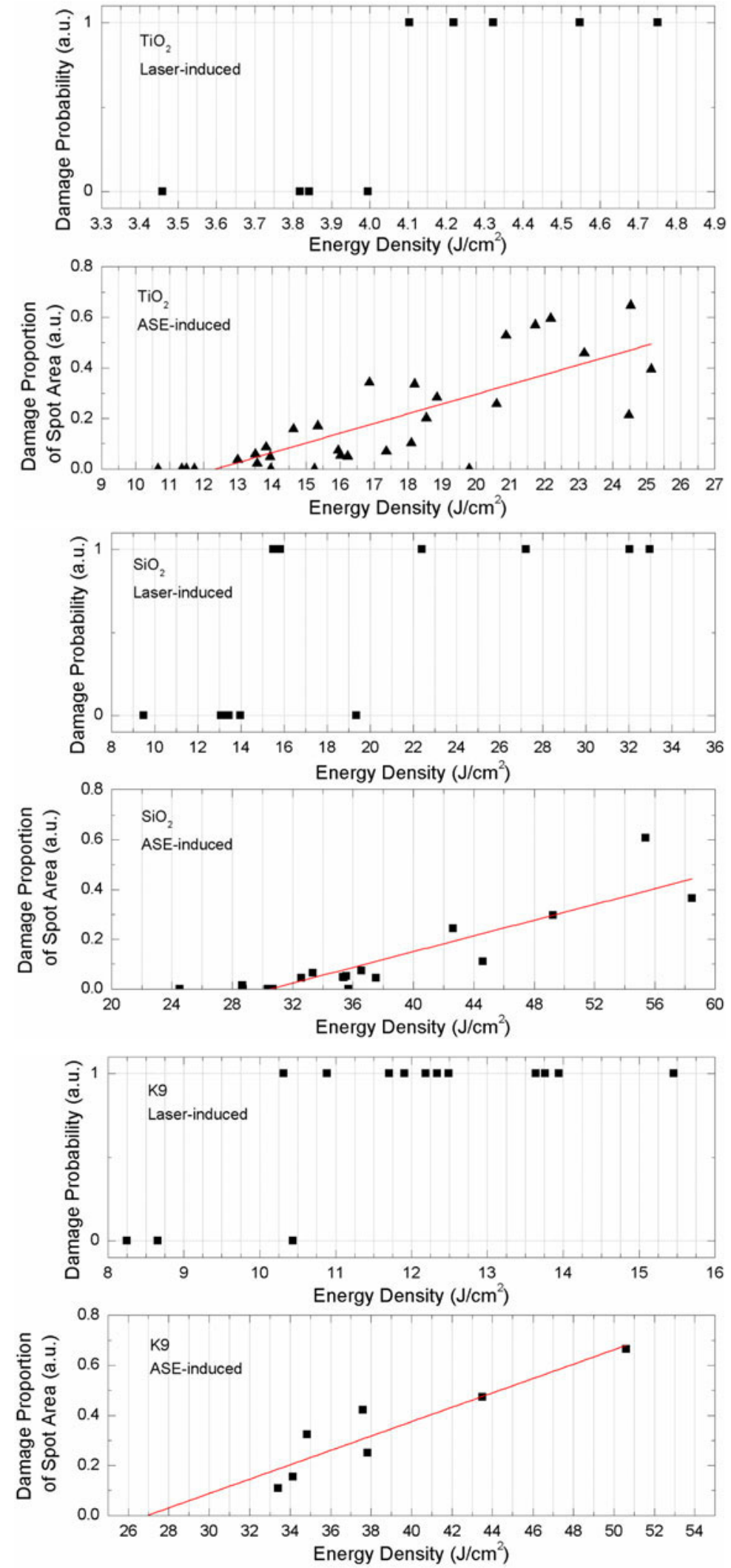

Figure 6. Damage threshold nonlinearly fitted using ASE and a laser as the test source, respectively. 
Hence, the diffraction patterns of each frequency component overlap and the nonuniformity is quickly smoothed out to a certain extent during the duration of pulse. Thus, there is less noise modulation in the near field of the ASE. Using ASE as the damage threshold test source, nonuniformity of the irradiation intensity caused by interference, diffraction and other nonlinear coherent effects can be neglected and the defects of elements can be studied in isolation. Thus, the AIDT is generally higher than the LIDT. In our experiment, the size of the test spot may not be large enough, due to small size samples. For large size optical components, we can test with large size spots of ASE. More defects can thus be revealed, leading to an accurate evaluation of the damage threshold.

\section{Conclusions}

Compared with the intensity distribution of a laser, the near field of ASE is very smooth, with less spatial modulation in the near field, and fewer hot spots in the far field. Thus, the effect of beam nonuniformity can be ignored in carrying out the damage threshold test. The experimental results show that the AIDT is clearly higher than the LIDT, leading to a more precise evaluation of the damage threshold.

\section{References}

1. H. Bercego, P. R. Bouchut, L. Lamaignere, B. L. Garrec, and G. Raze, Proc. SPIE 5273, 312 (2004).

2. E. Hacker, H. Lauth, and P. Weibrodt, Proc. SPIE 2714, 316 (1996).

3. J. Hue, F. Y. Genin, S. M. Maricle, and M. R. Kozlowski, Proc. SPIE 2966, 451 (1997).

4. A. F. Stewart and A. H. Guenther, Appl. Opt. 23, 3741 (1984).

5. L. Lamaignère, Laser-induced damage density measurements of optical materials, in Advanced Topics in Measurements (InTech, 2012).

6. H. Nakano, T. Kanabe, K. Yagi, K. Tsubakimoto, M. Nakatsuka, and S. Nakai, Opt. Commun. 78, 123 (1990).

7. H. Nakano, K. Tsubakimoto, N. Miyanaga, M. Nakatsuka, T. Kanabe, H. Azechi, T. Jitsuno, and S. Nakai, J. Appl. Phys. 73, 2122 (1993). 